\title{
STUDY THE DIFFERENCE BETWEEN THE MEASUREMENT OF THE RADIATION DOSE OF MRI AND CT SCAN
}

\author{
Hashim Fathi Yaseen \\ Department of Medical Physics \\ Teacher university \\ University of Mosul \\ Al Majmoaa str., Mosul, Iraq, 41002 \\ Department of Medical Optometry \\ Al-Kitab University \\ Karabeg, Alton Kopru, Iraq \\ hashim958io@gmail.com
}

\begin{abstract}
Radiation dose or absorbed dose means that the amount of energy absorbed by human tissue, and accurately is the concentration of energy deposited in tissue, when exposure to ionizing radiation, effective dose is the dose that will achieve the desired effect its unit is (millisievert or mSv). Ionizing radiation used in computed tomography (CT) scans as well as the associated health risks are an ongoing and growing concern.

The aim of this study is to prove the use of X-rays and computed tomography with a small dose of ionizing radiation to produce images for medical examination.

An MRI scan uses powerful magnets and radio waves to create images instead of ionizing radiation. Therefore, you are not exposed to radiation when you have an MRI scan, unlike a CT scan or X-ray. In addition to reducing the examination time and cost.

Material and methods show that individual doses measured for a routine whole-body CT protocol are less than $20 \mathrm{mGy}$ for all parts of the body. Thus, we conclude that the measured doses of the external organs are higher than those in the internal organs under the same conditions that are exposed to radiation Knowing that MRI does not use radiation (such as X-rays or CT scans) there are more MRI machines being produced from several companies around the world.

Knowing that MRI does not use radiation (such as X-rays or CT scans) there are more MRI machines being produced from several companies around the world.

Result in this study, it was done evaluate the benefits of MRI and CT in diagnoses and treatment as well as for their side effects, at present and in the future.

Conclusions. We have also concluded that CT is specific in certain areas of the human body such as the lungs and abdominal area and identify the areas of bone fractures, and magnetic resonance (MRI) gives us excellent image of high-definition of the spinal cord, brain and membranes, and the areas of the joints with animated images and a three - dimensional and in a record time of 15-30 minutes.

Where the diagnosis could be re-diagnosed during the procedure and after the occurrence of any side effects, unlike the tomography, which is specified in certain cases mentioned earlier, and a period of time of 5-10 minutes and cannot be returned except in special cases forced to have the effect of radiation. The final conclusion of our work, which is not only important in diagnosing, is that in the case of $\mathrm{CT}$, there are no disturbing sounds and the presence of an area in which the patient can settle, unlike magnetic resonance imaging, Where the annoying resonance sounds are added to the narrowness and smallness of the place where the patient is located, but they are not considered to be of medical importance.
\end{abstract}

Keywords: MRI, CT, absorbed dose, effective dose, mSv, processional, frequency, RF.

DOI: $10.21303 / 2504-5679.2022 .002281$

\section{Introduction}

In this research, first we will discuss the basic principles behind the physics of magnetic resonance imaging (MRI). Magnetic Resonance Imaging (MRI) is a non-invasive way to view organs, tissues, bones, and other structures inside the body. It uses strong magnetic fields and radio waves to produce internal images of the body. Unlike X-ray and CT scans, MRI machines produce cross sectional, 3D images of the body without using the use of radiation. Doctors often 
use MRI to view parts of the body that are hard to see with other imaging techniques such as x-rays, ultrasound, or computed tomography (CT) scans. MR imaging is used to diagnose many different types of diseases including heart and vascular disease, stroke, muscle and skeletal (bone) disorders and cancer $[1,2]$. The availability of higher resolution images from MRI means that these primary tumours can be found more easily, and patients' breasts can be conserved [3]

Second in case of CT system consists of an X-ray source, a rotary table, an X-ray detector and a data processing unit for computation, visualization and data analysis of measurement results (Fig. 1).

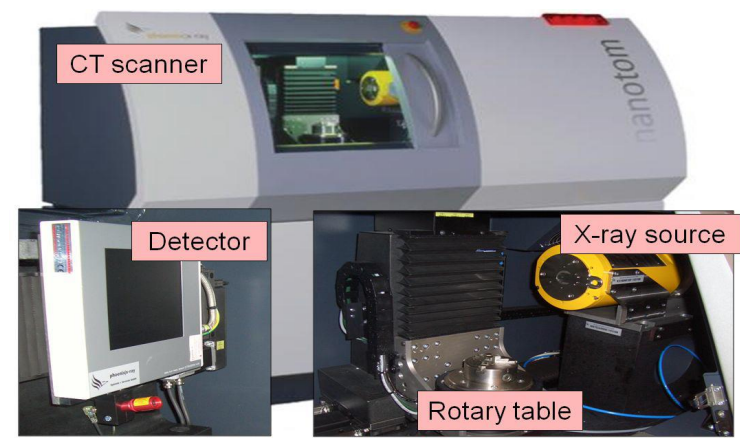

Fig. 1. An X-ray detector and a data processing unit for computation

In principle, $\mathrm{CT}$ creates cross section images by projecting a beam of emitted photons through one plane of an object from defined angle positions performing one revolution As the X-rays (emitted photons) pass through the object $\hat{\mathrm{A}}$, some of them are absorbed $\hat{\mathrm{A}}$, some are scattered $\hat{\mathrm{A}}$, and some are transmitted. The process of X-ray intensity reduction, involving just those $\mathrm{X}$-rays which are scattered or absorbed, is called attenuation. X-rays which are attenuated due to the interactions with the object do not reach the X-ray detector. Photons transmitted through the object at each angle are collected on the detector and visualized by computer, creating a complete reconstruction of the scanned object. The $3 \mathrm{D}$ grey value data structure gained in this way represents the electron density distribution in the measured object [4].

\subsection{Principles of $\mathrm{CT}$ scan and MRI}

Computed Tomography (CT)

Computed Tomography is use X-ray technology is used for the purpose of detecting various diseases and situations.

One of its advantages is known as fast, without pain, and does not cause side effects. It is characterized by accuracy in emergency situations where it can detect internal bleeding and internal wounds quickly enough to save the life of the patient. Its work as X-ray produces radiological images for the inside of the body to detect various diseases. The resulting images can be reconstructed from CT in multiple frames, so that they can be formatted on three-dimensional images, or can be converted to disc CD or DVD.

Give us the pictures of CT scan of the anatomy of the internal organs, bones, soft tissues and blood vessels more Details of the picture of the individual X-ray. For this, CT scans make it easier for us to diagnose, for example, cancer, heart disease, infectious diseases like tuberculosis, tuberculosis, and appendix.

Radiation risks of CT scan. Patients undergoing a CT examination typically receive organ doses on the order of tens of mGy. As a result, organ doses in CT are well below the threshold doses for the induction of deterministic effects such as skin burns and epilation, and the induction of eye cataract [4]. In the middle of the 20th century, when atmospheric testing of atomic weapons resulted in radioactive fallout on a worldwide scale, the genetic effects of radiation were deemed to be a major concern. Nowadays, however, any genetic risk is considered to be minor compared to the risk of carcinogenesis. Individual genetic risks are regarded as negligible, and any corresponding societal impact from diagnostic radiology is deemed to be very low. The principal concern at patient doses on the order of tens of mGy is the induction of fatal and nonfatal cancers [5]. 
So we prove that effective dose for chest from X-ray equal to $0.02 \mathrm{mSv}$, and from CT scan equal to 20mSv, (1000 times larger), for head (about 30 times larger), and for abdomen (about 37 times larger) $[6,7]$.

From the above we note the wide difference between X-ray and CT scan in terms of the affective dose which affecting the human body, so be careful to choose between X0ray and CT scan, and follow the necessary instructions to be mentioned in this research.

\section{Magnetic Resonance Imaging (MRI)}

Magnetic resonance imaging is a method of non-surgical examination for the purpose of examining and diagnosing the human body, especially soft tissues, bones and other internal organs of the body, which are of low intensity. In other words, they contain more water molecules than the rest of the body. In other words, contain hydrogen particles intensively and are the basis of the work of magnetic resonance.

The magnetic resonance system contains high magnetic fields starting from 1.5 tesla and more. Radiation waves are also used to stimulate the cells of the human body to be examined, where they are clearly visible and without the use of any radiation such as harmful X-rays. The advantages of magnetic resonance imaging, which doctors prefer to be clear, can be three - dimensional, easy to store on the computer or disk and re-use several times. During surgery, several images can be taken by MRI to identify the results of the operation without side effects $[8,9]$.

From the above the advantages of MRI can be summarized as shown in Fig. 2.
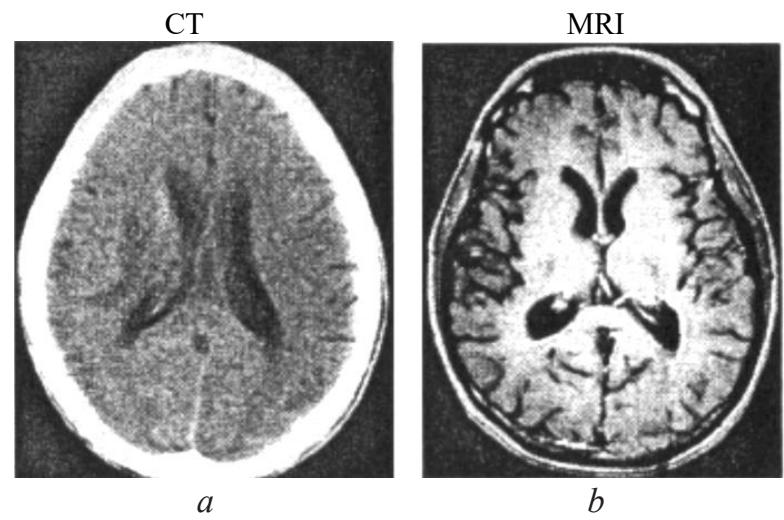

Fig. 2. Shows the differences between the last image of CT and MRI.

Clearly that from the figure shows:

1 - Obtain a clearly defined anatomical structure.

2 - Obtain a high contrast of soft tissue, which cannot be obtained from CT.

3 - Non-exposure to radiation as occurs in CT, because MRI uses non-harmful magnetic fields and radio waves $[8,10,11]$.

4 - MRI are expensive but they last longer if used correctly.

5 - The duration of the magnetic resonance scan may take up to 20 minutes and can be repeated several times without any radiological effect on the patient. Contrary to what happens in tomography $[12,13]$

Ionizing radiation used in computed tomography (CT) scans as well as the associated health risks are an ongoing and growing concern. The purpose of this study is to directly measure individual organ doses, and to assess how these measurements differ in different CT scan conditions [14, 15].

\section{Materials and Methods}

The materials used in this research are through the use of surveys, research and sources mentioned above in this research, and thus we deduced the relationship between the differences between the measurement of the radiation dose of MRI and CT SCAN, especially the amount of radiation dose used and absorbed by the patient's tissues through examining the patient with magnetic resonance devices, especially with CT SCAN. 
In this study, it was done evaluate the benefits of MRI and CT in diagnoses and treatment as well as for their side effects, at present and in the future. According to the international equivalent radiation dose standards (IAEA Safety Standards Series). [16, 17]

\section{Results}

Magnetic resonance imaging uses the large diffusion of hydrogen in the body in addition to the magnetic properties of the proton in the hydrogen atom. Whereas hydrogen atoms have a small magnetic field due to the rotation of the proton of this atom. For these reasons, Raymond Damadian hypothesized that cancerous cells can be distinguished from non-cancerous cells using magnetic resonance. Because the cancer cells contain more water and will appear in the MRI due to the increase in the number of hydrogen atoms present in the additional amount of water. For example, doctors and researchers use a variety of neuroimaging tools to study the brain.

CT scans are tilted slices of X-ray that show the density of brain structures. Whereas an MRI uses changes in electrically charged particles in a magnetic field to create images of the brain. That is, both techniques are more accurate than ordinary X-rays, and they can help detect many brain problems and diseases. In addition, these images also help doctors identify areas of the brain associated with different behaviours.

While a form of magnetic resonance imaging known as functional magnetic resonance imaging (fMRI), which is considered the most advanced of neuroimaging techniques, has emerged over the past years. So that it is his duty is to track the functional MRI of changes in blood flow and oxygen levels to indicate neural activity.

When a specific area of the brain is more active, it consumes more oxygen, which leads to increased blood flow. Thus, fMRI was used to provide a clear knowledge of cognitive functions in different brain regions, such as labelling areas related to visual perception, language or memory. Thus, fMRI researchers and doctors were able to identify and characterize brain functions at the level of neurological processes.

Although this might be confusing at times, it seems to be unavoidable. In any case, we'll try to keep it straight-forward.

\section{Discussions}

Magnetic resonance imaging (MRI) in monitoring brain development is well-defined. Magnetic resonance imaging (MRI) has demonstrated the ability to assess fetal and neonatal neurological conditions due to its unprecedented sensitivity and in vivo tissue contrast. With the help of magnetic resonance imaging, improved anatomical details and better sensitivity are revealed in detecting growth anomalies that were not identified in prenatal ultrasound.

The title of our research Study the difference between the measurement of the radiation dose of MRI and CT has benefits as can be seen from the following Table $\mathbf{1}$.

Table 1

Difference between the measurement of the radiation dose of MRI and CT

\begin{tabular}{ccc}
\hline MRI System & CT System & Function \\
\hline Principle of magnetic resonance & Absorption of radiation & Principle of imaging \\
No & Yes & Exposure of radiation \\
No & Influence by bone, air \\
Excellent for scientific information & Good for physical information & Tissue resolution \\
Easy for 2D\&3D & Difficult only for 2D & Dynamic diagnostic information 2D, 3D \\
Arbitrary section plane & Transverse plane & Image plane \\
Spinal cord, brand junction, etc. & Lung, abdomen, bone, etc. & Area of diagnoses \\
From 15 to 30 minutes & Between 5 and 10 minutes & Duration of diagnosis \\
The noise of the resonance sound is rather & Relatively quiet & Noise during diagnosis, voice or fear factor \\
annoying & for small space
\end{tabular}


We concluded from this research through the previous data and the table that CT give us partial information is almost few in the place with MRI, which gives accurate texture information through images 2D and 3D, as well as images of the longitudinal and cross section.

Study limitations. In this research, MRI cannot always distinguish malignant tumors or benign diseases in general, which may lead to false positive results. It can also be said that the MRI is not painful, but the patient must remain in a closed device, which may be a problem for patients who suffer from claustrophobia, so the patient is provided with headphones during the examination. Computerized tomography uses high-energy X-rays that are harmful to health, and CT scans are more common and less expensive.

Prospects for further research. In this research, we were able to prove that both MRI and CT scans are useful in diagnosing the internal organs of the body. However, a CT scan is faster and can provide clear images of tissues, organs, and skeletal structures. Whereas MRI displays highly subtle anatomical medical images that help doctors determine if there are abnormal tissues inside the body. That is, the MRI images are more detailed.

\section{Conclusion}

We have also concluded that $\mathrm{CT}$ is specific in certain areas of the human body such as the lungs and abdominal area and identify the areas of bone fractures, and magnetic resonance (MRI) gives us excellent image of high-definition of the spinal cord, brain and membranes, and the areas of the joints with animated images and a three - dimensional and in a record time of 15-30 minutes.

Where the diagnosis can be re-diagnosed during the procedure and after the occurrence of any side effects, unlike the tomography, which is specified in certain cases mentioned earlier, and a period of time of 5-10 minutes and cannot be returned except in special cases forced to have the effect of radiation. The final conclusion of our work, which is not only important in diagnosing, is that in the case of CT, there are no disturbing sounds and the presence of an area in which the patient can settle, unlike magnetic resonance imaging, Where the annoying resonance sounds are added to the narrowness and smallness of the place where the patient is located, but they are not considered to be of medical importance.

\section{Conflict of interests}

All hospitals used the same electronic program, to monitor the CT doses. The results may differ if the study collected data from hospitals that use other programs or do not use programs or use a GIGER counter device. The data may not represent all hospitals equally due to the different reagent equipment and electronic software in those countries.

\section{Financing}

The study was performed without financial support.

\section{Acknowledgments}

Many thanks to my family who supported me in all areas, and thanks to my university for providing me with scientific resources and free internet.

Many thanks to the professors who helped me in all areas, and thanks to my university for providing me with scientific resources and free internet.

\section{References}

[1] MRI of the Body (Chest, Abdomen, Pelvis) (2007). Radiology Info. Available at: https://www.beverlyhospital.org/ media/166251/bodymri.pdf

[2] Barentsz, J., Takahashi, S., Oyen, W., Mus, R., De Mulder, P., Reznek, R. et. al. (2006). Commonly Used Imaging Techniques for Diagnosis and Staging. Journal of Clinical Oncology, 24 (20), 3234-3244. doi: http://doi.org/10.1200/jco.2006.06.5946

[3] Buchanan, C. L., Morris, E. A., Dorn, P. L., Borgen, P. I., Van Zee, K. J. (2005). Utility of Breast Magnetic Resonance Imaging in Patients With Occult Primary Breast Cancer. Annals of Surgical Oncology, 12 (12), 1045-1053. doi: http://doi.org/10.1245/ aso.2005.03.520 
[4] Bartscher, M., Hilpert, U., Goebbels, J., Weidemann, G. (2007). Enhancement and Proof of Accuracy of Industrial Computed Tomography (CT) Measurements. CIRP Annals, 56 (1), 495-498. doi: http://doi.org/10.1016/j.cirp.2007.05.118

[5] Hall, E. J. (2000). Radiobiology for the radiologist. Philadelphia: Lippincott Williams \& Wilkins, 588.

[6] Bergen, R. V., Ryner, L. (2019). Assessing image artifacts from radiotherapy electromagnetic transponders with metal-artifact reduction imaging. Magnetic Resonance Imaging, 59, 137-142. doi: http://doi.org/10.1016/j.mri.2019.02.005

[7] Wanner, L., Ludwig, U., Hövener, J.-B., Nelson, K., Flügge, T. (2018). Magnetic resonance imaging - a diagnostic tool for postoperative evaluation of dental implants: a case report. Oral Surgery, Oral Medicine, Oral Pathology and Oral Radiology, 125 (4), e103-e107. doi: http://doi.org/10.1016/j.0ooo.2018.01.005

[8] Moolman, N., Mulla, F., Mdletshe, S. (2020). Radiographer knowledge and practice of paediatric radiation dose protocols in digital radiography in Gauteng. Radiography, 26 (2), 117-121. doi: http://doi.org/10.1016/j.radi.2019.09.006

[9] Hawarihewa, P. M., Satharasinghe, D., Amalaraj, T., Jeyasugiththan, J. (2021). An assessment of Sri Lankan radiographer's knowledge and awareness of radiation protection and imaging parameters related to patient dose and image quality in computed tomography (CT). Radiography. doi: http://doi.org/10.1016/j.radi.2021.10.010

[10] Garba, I., Zarb, F., McEntee, M. F., Fabri, S. G. (2020). Computed tomography diagnostic reference levels for adult brain, chest and abdominal examinations: A systematic review. Radiography, 27 (2), 673-681. doi: http://doi.org/10.1016/j.radi.2020.08.011

[11] Joseph Zira, D., Haruna Yahaya, T., Umar, M. S., Nkubli B, F., Chukwuemeka, N. C., Sidi, M. et. al. (2021). Clinical indicationbased diagnostic reference levels for paediatric head computed tomography examinations in Kano Metropolis, northwestern Nigeria. Radiography, 27 (2), 617-621. doi: http://doi.org/10.1016/j.radi.2020.11.021

[12] Liu, N., Johnson, K. J., Ma, C. X. (2018). Male Breast Cancer: An Updated Surveillance, Epidemiology, and End Results Data Analysis. Clinical Breast Cancer, 18 (5), e997-e1002. doi: http://doi.org/10.1016/j.clbc.2018.06.013

[13] Kubo, T. (2019). Vendor free basics of radiation dose reduction techniques for CT. European Journal of Radiology, 110, 14-21. doi: http://doi.org/10.1016/j.ejrad.2018.11.002

[14] Smith-Bindman, R., López-Solano, N., Miglioretti, D., Flynn, M., \& Seibert, J. A. (2020). Why Do Radiation Doses in CT Differ across Hospitals and Countries? Patient-Centered Outcomes Research Institute (PCORI). doi: http://doi.org/10.25302/07.2020. cd.13047043

[15] Radiation protection, $\mathrm{N}^{\circ}$ 185, European guidelines on diagnostic reference levels for paediatric imaging. European Commission (EC). Luxembourg: Publications Office of the European Union, 122.

[16] Mohd Tap, N. H., Jaafar Sidek, M. A., Mohd Ridzwan, S. F., Selvarajah, S. E. et. al. (2018). Computed Tomography Dose in Paediatric Care: Simple Dose Estimation Using Dose Length Product Conversion Coefficients. Malaysian Journal of Medical Sciences, 25 (4), 82-91. doi: http://doi.org/10.21315/mjms2018.25.4.8

[17] Saeed, M. K. (2020). A comparison of the CT-dosimetry software packages based on stylized and boundary representation phantoms. Radiography, 26 (4), e214-e222. doi: http://doi.org/10.1016/j.radi.2020.02.011

Received date 23.11.2021

(C) The Author(s) 2022

Accepted date 24.01.2022

This is an open access article

Published date 31.01.2022

under the Creative Commons CC BY license

How to cite: Yaseen, H. F. (2022). Study the difference between the measurement of the radiation dose of MRI and CT scan. EUREKA: Health Sciences, 1, 63-68. doi: http://doi.org/10.21303/2504-5679.2022.002281 